

\section{NOTICE}

This report was prepared as an account of work sponsored by an agency of the United States Government. Neither the United States Government nor any agency thereof, nor any of their employees, makes any warranty, express or implied, or assumes any legal liability or responsibility for the accuracy, completeness, or usefulness of any information, apparatus, product, or process disclosed, or represents that its use would not infringe privately owned rights. Reference herein to any specific commercial produce, process, or service by trade name, trademark, manufacturer, or otherwise, does not necessarily constitute or imply its endorsement, recommendation, or favoring by the United States Government or any agency thereof. The views and opinions of authors expressed herein do not necessarily state or reflect those of the United States Government or any agency thereof.

\section{NOTICE}

This report has been reproduced directly from the best available copy.

Available to DOE and DOE contractors from the:

Office of Scientific and Technical Information

P.O. Box 62

Oak Ridge, TN 37831;

Prices available from (615) 576-8401.

Available to the public from the:

National Technical Information Service

U.S. Department of Commerce

5285 Port Royal Road

Springfield, Virginia 22161

703-487-4650 


\title{
A UNIFIED THEORY OF RESONANT EXCITATION OF KINETIC BALLOONING MODES BY ENERGETIC IONS/ALPHA PARTICLES IN TOKAMAKS
}

\author{
H. Biglari and L. Chen \\ Plasma Physics Laboratory, Princeton University, P. O. Box 451, Princeton, NJ 08543
}

PACS Nos. 52.35.-g, 52.35.Qz, 52.35.Bj, 52.5.Fa

A complete theory of wave-particle interactions is presented whereby both circulating and trapped energetic ions can destabilize kinetic ballooning modes in tokamaks. Four qualitatively different types of resonances, involving waveprecessional drift, wave-transit, wave-bounce, and precessional drift-bounce interactions, are identified, and the destabilization potential of each is assessed. For a characteristic slowing-down distribution function, the dominant interaction is that which taps those resonant ions with the highest energy. Implications of the theory for present and future generation fusion experiments are discussed. 
The importance of energetic particle dynamics in present and future-generation tokamak devices stems not only from their ubiquitous role in heating the plasma to thermonuclear iemperatures (e.g., auxiliary beam ions, RF-produced fast ion tails), but also in self-sustaining the plasma state in the ignition regime (through fusion product alpha particles). Over the past decade, observations of magnetohydrodynamic (MHD) fluctuations associated with high-power auxiliary heating experiments ${ }^{1-4}$ and consequent loss of energetic ions has spurred considerable theoretical activity on how the stability of bulk plasma. MHD modes is influenced by the presence of energetic particles. The emergent physical picture has been that while suprathermal particles can have a stabilizing influence on normal MHD modes when the mode frequency is strongly off-resonant with the hot particle precessional drift frequency $\left(\omega \ll \omega_{d h}\right),{ }^{5}$ they become destabilizing when the wave structure begins to resonate with either the precessional drift motion of the hot particle banana center $\left(\omega \simeq \bar{\omega}_{d h}\right)^{6,7}$ or the transit motion of circulating hot ions $\left(\omega \simeq \omega_{t h}\right)^{8}$ In this Letter, we complete the picture and show that two other types of resonances, one involving wave-bounce interactions and the other magnetic drift-bounce interactions, are also possible and indeed dominant for trapped energetic ions. Our formulation thus places all types of resonant wave-particle interactions involving circulating and trapped energetic ions on the same footing, allowing us to discuss their relative importance with respect to different types of MHD fluctuations.

The plasma is taken to consist of two components: a warm core ( ' $c$ '), which is treated using an ideal MHD description (thus, there is no parallel electric field perturbation, i.e., $\delta E_{\|}=0$, which in turn couples the electrostatic potential $\delta \phi$, to the parallel vector potential $\delta A_{\|}$), and a tenuous, highly energetic component ( ' $h$ '), which is dealt with using gyrokinetic formalism. We assume the plasma lies in the first stability regime with respect to ballooning modes, and adopt the formal ordering $\beta_{c} \sim \epsilon, \beta_{h} / \beta_{c} \sim \epsilon$ (this assumption is easily relaxed), $n_{h} / n_{c} \sim \epsilon^{3}$, and $T_{c} / T_{h} \sim \epsilon^{2}$, where $\epsilon=r / R \ll 1$ is the local inverse aspect ratio. Standard manipulations starting with the continuity and momentum equations then yield the following eigenmode equation:

$$
\begin{aligned}
\mathbf{B} \cdot \nabla \frac{1}{B^{2}} \mathbf{B} \cdot \nabla \nabla^{2} \delta \phi & +\frac{\omega\left(\omega-\omega_{* i p}\right)}{v_{A}^{2}} \nabla^{2} \delta \phi \\
& -\frac{8 \pi p_{c}^{\prime}}{B^{2}} k_{\vartheta} \boldsymbol{\kappa} \times \hat{\mathbf{e}}_{\|} \cdot \nabla \delta \phi+\frac{4 \pi}{B^{2}} \frac{\omega}{c} \boldsymbol{\kappa} \times \mathbf{B} \cdot \nabla\left(\delta p_{\| h}+\delta p_{\perp h}\right)=0,
\end{aligned}
$$

where $\left(\delta p_{\| h}, \delta p_{\perp h}\right)=m_{h} \int d^{3} v\left(v_{\|}^{2}, v_{\perp}^{2} / 2\right) \delta F_{h}$ are the parallel and perpendicular components of the hot particle pressure tensor, $\omega_{* i p}=k_{\vartheta} \rho_{i} v_{t i} / L_{p i}, L_{p i}^{-1}=-d \ln p_{i} / d r, v_{A}^{2}=$ $B^{2} / 4 \pi m_{i} n_{i}$, and $\boldsymbol{\kappa}=\hat{\mathbf{e}}_{\|} \cdot \nabla \hat{\mathbf{e}}_{\|}$is the magnetic curvature. The first term in Eq. (1) is the shear Alfvén field line bending term, the second is associated with the ion polarization drift and incomplete charge cancellation between electrons and ions, the third is the convective core pressure ballooning drive (pressure weighting of unfavorable curvature), while the last term is the hot particle pressure drive. The hot particle distribution function is given by $\delta F_{h}=-\left(e_{h} \hat{\omega}_{* h} / m_{h} \omega\right) \delta \phi+\delta g_{h}$, where $\hat{\omega}_{* h}=\left(\mathbf{k} \times \hat{\mathbf{e}}_{\|} / \Omega_{h}\right) \cdot \nabla \ln F_{0 h}$, where $F_{0 h}$ is the equilibrium hot ion distribution function. The first term is the convective hot ion 
contribution (which in our ordering is subdominant to the core pressure drive), while the second is the hot particle compressional contribution which drives the instability. The latter satisfies the equation

$$
m_{h}\left[v_{\|} \partial_{l}-i\left(\omega-\omega_{d h}\right)\right] \delta g_{h}=Q_{h} e_{h} i \omega_{d h} \delta \phi,
$$

where $Q_{h}=\left(\hat{\omega}_{* h} / \omega+\partial_{E}\right) F_{0 k}$, and $l$ denotes the coordinate along the field line. The essence of the instability can be read off directly from Eq. (2). The lhs of Eq. (2), which is a balance between the bounce/transit frequency, the wave frequency, and the precessional drift frequency, characterizes the nature of the wave-particle interaction. $Q_{h}$ represents information about the free energy available to drive the instability and contains contributions from both configuration space and velocity space gradients. Finally, $e_{h} i \omega_{d h} \delta \phi=-e_{h} \mathbf{v}_{d h} \cdot \delta \mathbf{E}_{\perp}$ represents the rate at which the precessional kinetic energy of the energetic particles gets transformed into electromagnetic energy. The rate of this energy exchange mechanism is spatially varying, being more efficient in some regions of space and less so in others. Since the efficiency depends on the spatial structure of the mode, it is necessary to specify the type of MHD mode under consideration before proceeding further. Our focus in this Letter is on kinetic ballooning modes (KBM), ${ }^{9}$ which manifest themselves locally close to the first ideal ballooning stability boundary. In this context, it is important to point out that even if the plasma is globally below the first stability boundary, locally it can be close to it in regions of weak magnetic shear, such as exist close to the plasma center. This class of modes deserves special scrutiny because they are discrete in character $\left(\omega_{r} \simeq \omega_{\text {*ip }}\right.$, where $\omega_{r}$ is the real part of the frequency), and hence are much more susceptible to instability than modes in the Alfvén continuum. Indeed, it is very difficult to agitate a mode in the MHD continuum, since any such excitation is strongly damped through phase-mixing with all the other modes in the continuum. We will return to this point later.

We now outline the derivation leading to the dispersion relation, placing special emphasis on the physical ideas that have gone into the calculation. Adopting a shifted-circle, magnetic surface equilibrium model, ${ }^{10}$ and transforming to ballooning cooidinates, we arrive at the following integro-differential eigenmode equation:

$$
\frac{d}{d \vartheta}\left(1+\hat{s}^{2} \vartheta^{2}\right) \frac{d}{d \vartheta} \delta \phi+\left[\frac{\omega\left(\omega-\omega_{* i p}\right)}{\omega_{A}^{2}}-\delta W_{u}\right]\left(1+\hat{s}^{2} \vartheta^{2}\right) \delta \phi+\Delta g(\vartheta) \delta \phi+\delta W_{h, t}(\vartheta)=0
$$

where

$$
\begin{gathered}
\delta W_{u}=2 \pi q^{2} \sum_{j=h, i} \frac{m_{j}}{B^{2}} \int_{u} d^{3} v\left(v_{\|}^{2}+\frac{v_{\perp}^{2}}{2}\right)^{2} \frac{\omega\left(\hat{\omega}_{* j}+\omega \partial_{E}\right) F_{0 j}}{\omega_{t}^{2}-\omega^{2}}, \\
\delta W_{h, t}=4 \pi q^{2} \frac{m_{h}}{B^{2}}\left(\frac{\Omega_{h} R}{k_{\vartheta}}\right)^{2} \sum_{p=-\infty}^{\infty} \int_{t r} d^{3} v \frac{\left(\hat{\omega}_{* h}+\omega \partial_{E}\right) F_{0 h}}{\omega-\bar{\omega}_{d h}-p \omega_{b h}} \omega_{d h}(\vartheta) \cos I_{-\vartheta_{t}}^{\vartheta} \frac{\omega_{d h}(\vartheta) \cos I_{-\vartheta_{\imath}}^{\vartheta} \delta \phi}{} \\
I_{\vartheta_{a}}^{\vartheta_{b}}=\int_{\vartheta}^{\vartheta_{b}} d l\left[\omega-\omega_{d h}(\vartheta)\right] /\left|v_{\|}\right|, \Delta=\beta^{\prime} q^{2} R \text { is the Shafranov shift, } g(\vartheta)=\cos \vartheta+\hat{s} \vartheta \sin \vartheta- \\
\Delta \sin ^{2} \vartheta-\epsilon\left(1-q^{-2}\right), \hat{s}=r q^{\prime} / q, \omega_{t}=v_{\|} / q R_{0} \text { is the transit frequency, } \omega_{b}=2 \pi / \tau_{b}=
\end{gathered}
$$


$2 \pi\left[\oint_{-\vartheta_{t}}^{+\vartheta_{t}}\left(d l /\left|v_{\|}\right|\right]^{-1} \simeq \epsilon^{1 / 2}(2 E)^{1 / 2} / q R_{0}\right.$ is the bounce frequency, the subscript ' $t$ ' (' $u$ ') denotes (un)trapped particles, and an overhead bar denotes a bounce average, $\overline{(\cdots)}=$ $\oint_{-\vartheta_{t}}^{+\vartheta_{t}}\left(d l /\left|v_{\|}\right|\right)(\cdots) / \tau_{b}$, where $\vartheta_{t}$ is the banana turning point. $\delta W_{u}$ represents wave-transit (hot and core) ion resonances for both signs of $v_{\|}$, while $\delta W_{h, t}$ represents wave-precessional drift-bounce harmonic resonances. In particular, the $p=0$ contribution picks out the usual wave-precessional drift resonance $\omega=\bar{\omega}_{d h},{ }^{6,7}$ and the $p= \pm 1$ contribution picks out the fundamental wave-precession-bounce resonance. We now determine the spatial locus where wave-particle interaction is maximized. For the problem at hand, ballooning space naturally divides itself into two regions: $a$ ) an ideal region for small $\vartheta$, and $b$ ) an inertial region for large $\vartheta$. Trapped energetic ions contribute symmetrically about $2 \pi$-periodic regions where $-\vartheta_{t}+2 \pi n \leq \vartheta \leq \vartheta_{t}+2 \pi n, n=0,1,2, \ldots$ Now, the precessional drift frequency, to leading order, is composed of two contributions: a periodic contribution, $\omega_{d h, \vartheta} \equiv k_{\vartheta} v_{d h, \vartheta} \propto \cos \vartheta$, and a secular contribution given by $\omega_{d h, r} \equiv k_{r} v_{d h, r} \propto \vartheta \sin \vartheta$. For ballooning modes, $\delta \phi(\vartheta)$ has even symmetry and is flutelike in structure for large $\vartheta$. When the wave can only resonate with the slow precessional motion of the hot particle banana center $(p=0)$, the rapid bounce motion of the energetic trapped ions averages out the up-down asymmetric (odd) wave-particle interaction associated with the secular component of $\mathbf{v}_{d h, r} \cdot \delta \mathbf{E}_{r}$. Since it picks out the nonsecular component, the $p=0$ resonance thus contributes dominantly to the ideal region. For the $p= \pm 1$ resonance, on the other hand, $\omega_{d h, r}$ makes the dominant contribution. Physically, in the frame of reference of the bounce motion, $\mathbf{v}_{d r} \cdot \delta \mathbf{E}_{r}$ is no longer averaged out and since $k_{r} \gg k_{\vartheta}$, this contribution dominates over $\mathbf{v}_{d h, \vartheta} \cdot \mathbf{E}_{\vartheta}$. By picking out the secular component, the $p= \pm 1$ resonance thus contributes primarily to the inertial region. We are then lead to the following dispersion relation:

$$
\begin{gathered}
-i\left[\frac{\omega\left(\omega-\omega_{* i p}\right)}{\omega_{A}^{2}}-\delta W_{h, u}-\Gamma_{I L D}-\delta W_{h, t}^{b}\right]^{1 / 2}+\delta W_{f}+\delta W_{h, t}^{p}=0, \\
\operatorname{Im} \delta W_{h, u} \simeq \frac{\pi^{2}}{2^{5 / 2}} q^{2} \beta_{h} \frac{\omega_{r}}{\omega_{t m}} \sum_{\sigma= \pm} \int_{0}^{1} d \bar{v}_{\|} \int_{0}^{\bar{v}_{\| l} / \sqrt{2 \epsilon}} d \bar{v}_{\perp} \bar{v}_{\perp}\left(\bar{v}_{\|}^{2}+\frac{\bar{v}_{\perp}^{2}}{2}\right)^{2} \\
\left(\frac{\omega_{* p m}}{\omega_{r}}-\frac{3}{2 \bar{E}}\right) \bar{E}^{-3 / 2} \delta\left(\sigma \bar{v}_{\|}-\frac{\omega_{r}}{\omega_{t m}}\right), \\
\operatorname{Im} \delta W_{h, t}^{b} \simeq \frac{\pi}{2} q^{2} \beta_{h} \epsilon^{1 / 2} \omega_{r} \int_{0}^{1} d \bar{E} \bar{E}\left(\frac{\omega_{* p m}}{\omega_{r}}-\frac{3}{2 \bar{E}}\right) \delta\left( \pm \omega_{b m} \bar{E}^{1 / 2}+\bar{\omega}_{d m} \bar{E}-\omega_{r}\right),
\end{gathered}
$$

where we have separated out the $p=0$ and $p= \pm 1$ contributions (higher bounce harmonic resonances make a progressively smaller contribution to the instability and can be neglected), and specialized to the case of an isotropic, hot ion slowing-down distribution function, $F_{0 h}=\left(B^{2} \beta_{h} / 2^{9 / 2} m_{h} \pi^{2} E_{m}\right) E^{-3 / 2}, E_{c r} \ll E \leq E_{m}\left[E_{m}\right.$ in the maximum (birth) energy and $E_{c r}$ is the energy at which the ions change from slowing down on the electrons to pitch angle scattering off the bulk ions]. ${ }^{11}$ In Eq. (4), $\delta W_{f}$ and $\delta W_{h, t}^{p}$ are, respectively, the fluid $\mathrm{MHD}$ and precessional drift contribution to the dispersion relation, 
and are given in Ref. 7 , while $\Gamma_{I L D}$ is the stabilizing contribution of ion Landau damping, which we shall discuss momentarily. ${ }^{14}$ In Eq. (5), frequencies decorated with a subscript ' $m$ ' denote their value at $E=E_{m}$. Several observations can be made about Eq. (5). First, instability ensues when the energetic ion pressure gradient is sufficiently steep as to overcome the stabilizing influence of velocity-space gradients. Second, the unstable mode rotates in the same direction as the energetic ions. Third, it is those resonant ions with the highest energy that make the dominant contribution to the instability. This is due to i) the energy (pressure) weighting of the velocity integrals, and ii) the fact that velocity-space stabilization increases with decreasing energy $\left(\propto E^{-1}\right)$. This is a unique characteristic of slowing-down distributions and in distinct contrast, for example, to what one would conclude for a Maxwellian distribution function, suggesting that there are important qualitative differences between the behavior of RF-produced energetic ions on the one hand, and NB energetic ions or alpha particles on the other.

Analysis of the dispersion relation Eq. (4) manifests for:r qualitatively different types of resonances:

i) Wave-precessional drift $(\mathrm{P})$ resonance $\left(\omega=\bar{\omega}_{d h}\right)$ : Here, energy is exhanged, flux invariance is broken, but the action invariant is preserved. Thus, the hot ion banana center moves out radially as it yields energy to the MHD wave. The result is an instability below the ideal ballooning stability boundary $\left(\delta W_{f}>0\right)$ when the hot particle beta exceeds continuum damping: ${ }^{7} \omega_{r} \simeq \omega_{A} \delta W_{f} \gg \omega_{* i p}\left(\delta W_{f} \sim \epsilon\right), \gamma \simeq \bar{\omega}_{d m}\left(\beta_{h}-\beta_{h, c r}\right)$, where $\beta_{h, c r} \simeq 2 \hat{s} \bar{\omega}_{d m} / \pi^{2} q^{2} \omega_{A}$.

ii) Wave-transit ion $(\mathrm{T})$ resonance $\left(\omega=\omega_{t h}\right)$ : In this case, energy is exchanged, and both action and flux invariants are broken. One obtains a 'thresholdless' (within the ideal MHD context) instability near ideal ballooning marginal stability $\left(\delta W_{f} \simeq 0\right)$, with real frequency given by $\omega_{r} \simeq \omega_{* i p}$ and growth rate

$$
\frac{\gamma}{\omega_{* i p}} \simeq \frac{\pi^{2}}{16} q^{2} \epsilon^{3 / 2} \beta_{h} \frac{\omega_{A}^{2}}{\omega_{t m}^{2}}\left(\frac{\omega_{* i p} \omega_{* p m}}{3 \omega_{t m}{ }^{2}}-2 \epsilon\right) .
$$

An upper bound on the instability can be obtained from the validity criterion $\omega_{t m}>$ $\bar{\omega}_{d m} E_{\text {res }} / 2 \epsilon$, where $E_{\text {res }} / E_{m} \simeq\left(\omega_{* i p} / \omega_{t m}\right)^{2} /(2 \epsilon) \ll 1$ is the resonant energy (corresponding to marginally-circulating energetic ions):

$$
\gamma_{\max } \simeq \frac{\pi^{2}}{8} q \frac{n_{h, u}}{n_{c}}\left(\frac{R}{L_{p i}}\right)^{1 / 2} \frac{v_{t i}}{L_{p h}}\left(1-\frac{L_{p h}}{R}\right)
$$

where $n_{c}$ and $n_{h, u}$ are the densities of the core particles and untrapped energetic ions, respectively.

iii) Wave-bounce (B) resonance $\left(\omega=\omega_{b h}\right)$ : This interaction is the trapped-particle analogue of the $\mathrm{T}$-type resonance. In this instance, the validity criterion is $\left.k \rho_{b h}\right|_{E_{\text {res }}}=1$, where $\rho_{b h}$ is the hot ion banana width, and $E_{\text {res }} / E_{m}=\left(\omega_{* i p} / \omega_{b m}\right)^{2}$, which is the same as for the T-resonance. The growth rate is the same as Eq. (7), except that $n_{h, t} \simeq \epsilon^{1 / 2} n_{h, u}<$ $n_{h, u}$ replaces $n_{h, u}$. 
iv) Precessional drift-bounce (PB) resonance $\left(\omega_{b h} \simeq \bar{\omega}_{d h} \gg \omega\right)$ : Here, energy is conserved, but both flux and action invariants are broken. Thus, although the pitch angle remains the same, the turning points move out vertically and the banana center moves out radially. The result is a 'thresholdless' instability near ideal ballooning marginality, with characteristics much akin to magnetic ripple-induced particle loss. Moreover, since $E_{\text {res }} / E_{m} \simeq\left(\omega_{b m} / \bar{\omega}_{d m}\right)^{2} \simeq 1$, the most energetic ions are tapped by this resonance, making it the most serious type of instability among the four. For a slowing-down distribution function, the instability is dominated by the longest wavelengths, $k_{\vartheta} \rho_{i} \simeq\left(\epsilon T_{c} / T_{h}\right)^{1 / 2} / q \ll 1$. At such long wavelengths, ion Landau damping, given by $\Gamma_{I L D} \simeq-i\left(\pi^{1 / 2} / 4\right) q^{2} \beta_{i}\left(\omega / \omega_{t i}\right) \exp \left(-\omega^{2} / \omega_{t i}^{2}\right)$, enters the problem prominently, and moves the discrete MHI) mode off the real frequency axis (in the absence of energetic particle destabilization) into the lower-half complex plane. There is then a low threshold ${ }^{15}$ that needs to be overcome for instability to set in. This qualitatively explains the result from kinetic codes ${ }^{12}$ that the local ballooning mode threshold is reduced in the presence of $\alpha$-particles. The frequency can then be calculated to be $\omega \simeq \exp (i \pi / 4)\left(\pi \beta_{h} \omega_{* p m} / \bar{\omega}_{d m}\right)^{1 / 2} q \omega_{b m} \omega_{A} / \bar{\omega}_{d m}$, with a maximum growth rate given by

$$
\gamma_{\max } \simeq\left(\frac{\pi}{2} \epsilon^{1 / 2} \beta_{h, t r} \frac{R}{L_{p h}}\right)^{1 / 2} q \omega_{A} .
$$

Comparing the last three instabilities, we find

$$
\frac{\gamma_{\max }^{P B}}{\gamma_{\max }^{B}} \sim \frac{\gamma_{\max }^{P B}}{\gamma_{\max }^{T}} \sim \frac{1}{q}\left(\frac{\epsilon^{1 / 2}}{2 \pi} \frac{\beta_{h}}{\beta_{c}} \frac{L_{p i} L_{p h}}{R^{2}}\right)^{1 / 2} \frac{n_{c}}{n_{h}} .
$$

For a Maxwellian distribution function, the instability would peak at intermediate wavelengths, resulting in smaller growth rates. En passant, we note that this type of resonance is extremely inefficient for circulating ions, since then $\omega_{d h}$ varies sinusoidally and the particles do not remain in resonance with the wave, but rather are constantly moving in and out of resonance.

We now discuss the implications of the theory for present and future-generation experiments, as well as the interpretation of older experiments in light of new results presented here. Consider first the high-frequency precursor to fishbone oscillations. ${ }^{1}$ While the interpretation of the fishbone itself in terms of precessional resonance destabilization of the internal kink mode ${ }^{6}$ appears quite credible and consistent with the experimental observations, the characterization of its high-frequency precursor oscillation by precessional resonant destabilization of ballooning modes $^{7}$ has been less compelling. The main reason is that the $\beta_{h}$ threshold for destabilization is essentially the same expression for both modes and scales linearly with the toroidal mode number $n$. The implication is that if both fluctuations emanate from the same region of the plasma, the fishbone instability $(n=1)$ should be triggered before its high-frequency precursor $(n \gg 1)$. This inconsistency is readily resolved within the context of the present theory. In the region near the plasma center, where the fishbone instability is believed to manifest itself, the $q$ profile is very flat, and hence the plasma is likely to be locally close to the first stability ballooning 
boundary. The drift-bounce resonance, which we have shown to be not only dominant over the precessional drift resonance, but also 'thresholdless' within the context of ideal MHD, is then a much more viable candidate to explain the high-frequency fishbone precursor oscillation. Furthermore, the parallel fishbone oscillations observed on $\mathrm{PBX},{ }^{2}$ car: be readily identified with the wave-transit resonance destabilization of KBM's. ${ }^{8}$ Turning next to recent experimental results of fast ion loss on DIII-D ${ }^{4}$ which has been advocated as tentative evidence for toroidal Alfvén eigenmode (TAE) fluctuations, ${ }^{13}$ we note that there is nothing in the observational evidence which precludes the simultaneous existence of both transit-resonantly destabilized KBM's and TAE oscillations. Indeed, the fact that $\omega_{* \text { *ip }}$ and $\omega_{T A E} \simeq v_{A} / 2 q R$ are roughly comparable in magnitude makes a clean separation of the two oscillations difficult in that experiment. Two possibilities which might shed light on this issue may be to do a toroidal magnetic field scan at fixed $q$, or a plasma current scan at fixed field. Alternatively, one can compare the scaling of the resonant parallel velocity as a function of the Alfvén velocity. Finally, we discuss the implications of the theory for alpha particles in DT plasmas. First note from Eq. (9) that there is a quantitative difference between NB-produced energetic ions and alpha particles, in that for the former, typically $n_{c} / n_{h} \lesssim 10$ and $\beta_{c} \sim \beta_{h}$, so that $\gamma_{\max }^{P B} / \gamma_{\max }^{B} \sim \gamma_{\max }^{P B} / \gamma_{\max }^{T} \sim 1$, but for the latter, $n_{c} / n_{h} \sim 10^{3}$ and $\beta_{h} / \beta_{c} \sim 0.1$, so that $\gamma_{\max }^{P B} / \gamma_{\max }^{B} \sim \gamma_{\max }^{P B} / \gamma_{\max }^{T} \gg 1$. Furthermore, the radial distribution of $\alpha$ particles is expected to be sharply peaked about the plasma center because of the sensitive dependence of the $\mathrm{D}-\mathrm{T}$ reaction cross section on temperature. Using typical parameters and profiles obtained from simulations of the proposed DT experiment on TFTR, ${ }^{16}$ we therefore expect that KBM destabilization by $\alpha$ particles will be maximized about the region $0.2 \lessgtr r / a \lesssim 0.3$, with typical toroidal mode numbers between 2 and 5 . Since the calculations presented in this Letter are for local modes, more nonlocal analysis is required to assess the lower limit of this range.

\section{ACKNOWLEDGMENTS}

This work was supported by the U. S. Department of Energy Contract \#DE-AC0276-CHO3073. 


\section{References}

${ }^{1}$ K. McGuire et al., Phys. Rev. Lett. 50, 89 (1983).

${ }^{2}$ W. W. Heidbrink et al., Phys. Rev. Lett. 57, 835 (1987).

${ }^{3}$ K.-L. Wong et al., Phys. Rev. Lett. 66, 1874 (1991).

${ }^{4}$ W. W. Hejubrink et al., Nucl. Fusion (in press).

${ }^{5}$ J. W. Connor et al., in Proc. 3rd Joint Varenna-Grenoble International Symposium on Heating in Toroidal Plasmas (CEC, Brussels, 1982) Vol. 1, p. 65; M. N. Rosenbluth et al., Phys. Rev. Lett. 51, 1967 (1983).

${ }^{6}$ L. Chen, R. W. White, and M. N. Rosenbluth, Phys. Rev. Lett. 52, 1122 (1984); H. Biglari and L. Chen, Phys. Fluids 29, 1760 (1986); B. Coppi and F. Porcelli, Phys. Rev. Lett. 57, 2277 (1986).

${ }^{7}$ J. Weiland and L. Chen, Phys. Fluids 28, 1359 (1985); H. Biglari and L. Chen, Phys. Fluids 29, 2960 (1986); ; D. Spong et al., Fusion Tech. 13, 428 (1988).

${ }^{8} \mathrm{~L}$. Chen, in Theory of Fusion Plasmas, Ed. J. Vaclavik, F. Troyon, and E. Sindoni (CRPP, Association EURATOM, 1988), p. 327.

${ }^{9}$ W. M. Tang, J. W. Connor, and R. J. Hastie, Nucl. Fusion 20, 1439 (1980).

${ }^{10}$ J. W. Connor, R. J. Hastie, J. B. Taylor, Phys. Rev. Lett. 40, 396 (1978).

${ }^{11}$ 'The numerical coefficient of the velocity space stabilization term would be $1 / 2$ instead of $3 / 2$ if the distribution function were mono-pitch angle (as in neutral beam injection, for example) instead of isotropic (as in fusion product alpha particles).

${ }^{12}$ G. Rewoldt, Phys. Fluids 31, 3727 (1988).

${ }^{13}$ C. Z. Cheng, L. Chen, and M. S. Chance, Ann. Phys. 161, 2i (1985); G. Y. Fu and J. W. Van Dam, Phys. Fluids B 1, 1949 (1989); C. Z. Cheng, Phys. Fluids B 2, 1427 (1990).

${ }^{14}$ Electron Landau damping can be shown to be smaller by a factor $v_{t i} / v_{t e} \ll 1$. The two-dimensional structure of the mode and the consequent possibility of continuum damping remains to be explored.

${ }^{15}$ The threshold is obtained by solving the transcendental dispersion relation. This, in general, must be obtained numerically, but for the very pessimistic (for instability) case of $\omega \sim \omega_{t i}$, is given roughly by $\beta_{h, c r} \lesssim\left(L_{p h} / R\right) \beta_{c}$.

${ }^{16}$ R. Budny et al., submitted to Nuclear Fusion. 


\section{EXTERNAL DISTRIBUTION IN ADDITION TO UC-420}

Dr. F. Paoloni, Univ. of Wollongong, AUSTRALIA

Prof. M.H. Bronnan, Univ. of Sydnoy, AUSTRALIA

Plasma Research Lab., Australian Nat. Univ., AUSTRALIA

Prof. I.R. Jones, Flinders Univ, AUSTRALIA

Prof. F. Cap, Inst. for Theoretical Physics, AUSTRIA

Prof. M. Heindler, Institut für Theoretische Physik, AUSTRIA

Prof. M. Goossens, Astronomisch Instituut, BELGIUM

Ecole Royale Militaire, Lab. de Phy. Plasmas, BELGIUM

Commission-European, DG. XII-Fusion Prog., BELGIUM

Prof. R. Bouciquie, Rijksuniversiteit Gent, BELGIUM

Dr. P.H. Sakanaka, Instituto Fisica, BRAZIL

Instituto De Pesquisas Espaciais-INPE, BRAZIL

Documents Office, Alomic Energy of Canada Lid., CANADA

Dr. M.P. Bechynskj, MPE Technologies, Inc., CANADA

Dr. H.M. Skaregard, Univ. of Sackatchewan, CANADA

Prof. J. Teichmann, Univ. of Montreal, CANADA

Prof. S.R. Sreenivasan, Univ. of Calgary, CANADA

Prof. T.W. Johnston, INRS-Energie, CANADA

Dr. R. Bolbon. Centre canadien de tusion magnátique, CANADA

Dr. C.R. Janies., Univ. of Alberta. CANADA

Dr. P. Lukac, Komenskeho Universzita, CZECHOSLOVAKIA

The Librarian, Cuthem Leboratory, ENGLAND

Library, R61, Rutherford Appleton Laboratory, ENGLAND

Mrs. S.A. Hutchinson, JET Library, ENGLAND

P. Mahonen, Univ. of Helsinki. FINLAND

C. Mouttet, Lab. de Physique des Milieux lonisés, FRANCE

J. Radet, CEN/CADARACHE - Bat 506, FRANCE

Ms. C. Rinni, Univ. of loamina, GREECE

Dr. T. Mual, Acedemy Bibliographic Ser., HONG KONG

Proprint Library. Hungarian Academy of Sci., HUNGARY

Dr. B. Das Gupta, Saha Inst. of Nuclear Physics, INDIA

Dr. P. Kaw, Inst. for Plasma Rosearch, IND'A

Dr. P. Rosenau, isreol Inst. of Technology, ISRAEL

Librarian, Intemational Center for Theo Physics, ITALY

Miss C. Lle Palo, Associazione EURATOMENEA, ITALY

Dr. G. Grosko, Istituto di Fisica del IJlasma, ITALY

Dr. H. Yamulo, Toshiba Res \& Dovel Center, JAPAN

Prof. I. Kewakami, Atomic Energy Res.Inst. JAPAN

Prof. K. Nishikawa, Hiroshima Univ., JAPAN
Director, Japan Atomic Energy Research Inst., JAPAN

Prot. S. Itoh, Kyushu Univ., JAPAN

Data and Planning Center, Nagoya Univ. JAPAN

Prof. S. Tanaka, Kyoto Univ., JAPAN

Library, Kyoto Univ., JAPAN

Prof. N. Inoue, Univ. of Tokyo, JAPAN

S. Mori, Technical Advisor, JAERI, JAPAN

O. Mitarai, Kumamoto Inst. of Technology, JAPAN

H. Jeong, Korea Advanced Energy Research Inst., KOREA

Prof. D.I. Choi, The Korea Adv. Inst. of Sci. \& Tech., KOREA

Prof. B.S. Liloy, Univ. of Waikato, NEW ZEALAND

Inst. of Plasma Physics, PEOPLE'S REPUBLIC OF CHINA

Librarian, Inst. of Physics, PEOPLE'S REPUBUC OF CHINA

Library, Tsinghua Univ., PEOPLE'S REPUBLIC OF CHINA

2. L, S.W. Inst Physics. PEOPLE'S REPUBLC OF CHINA

Prot. J.A.C. Cabral, Instituro Superior Tecnico. PORTUGAL

Dr. O. Potrus, AL I CUzA Univ., ROMANIA

Dr. J. de Villiers, Fusion Sudies, AEC, S. AFRICA

Prof. M.A. Hellberg. Univ. of Natal, S. AfRICA

C.I.E.M.A.T, Fusion Division Librery. SPAIN

Dr. L. Sionfio, Univ. of UMEA, SWEDEN

Library, Royed Inst. of Technology, SWEDEN

Prof. H. Wilhelmson, Chalmers Univ. of Tech., SWEDEN

Contro Phys. Des Plasmas, Ecole Polytoch, SWIZERLAND

Bibliothook, Inst. Voor Plasma-Fysica, THE NETHERLANDS

M. Durgut, Vice Chairmen, Middio East Tech. Univ., TURKEY

Dr. D.D. Ryutov, Siberian Branch of Academy of Sci., USSR

Dr. G.A. Eliseev, Kurchatov Inst. USSR

Librarian, The Ukr.SSR Academy of Sciences, USSR

Dr. L.M. Kovrizhnykh, Inst of General Physics, USSR

Kemforschungeanlage GmbH, Zentrabibliothek, W. GERMANY

Bibliothak, Inst. Fü Plasmaforschung, W. GERMANY

Prof. K. Schinder, Ruhr-Universitat Bochum, W. GERMANY

Dr. F. Wagner, (ASDEX), Max-Planck-Institut, W. GEAMANY

Librarian, Max-Planck-Institut, W. GERMANY

Prot. R.K. Janev, Inst. of Physics, YUgosLAVIA 

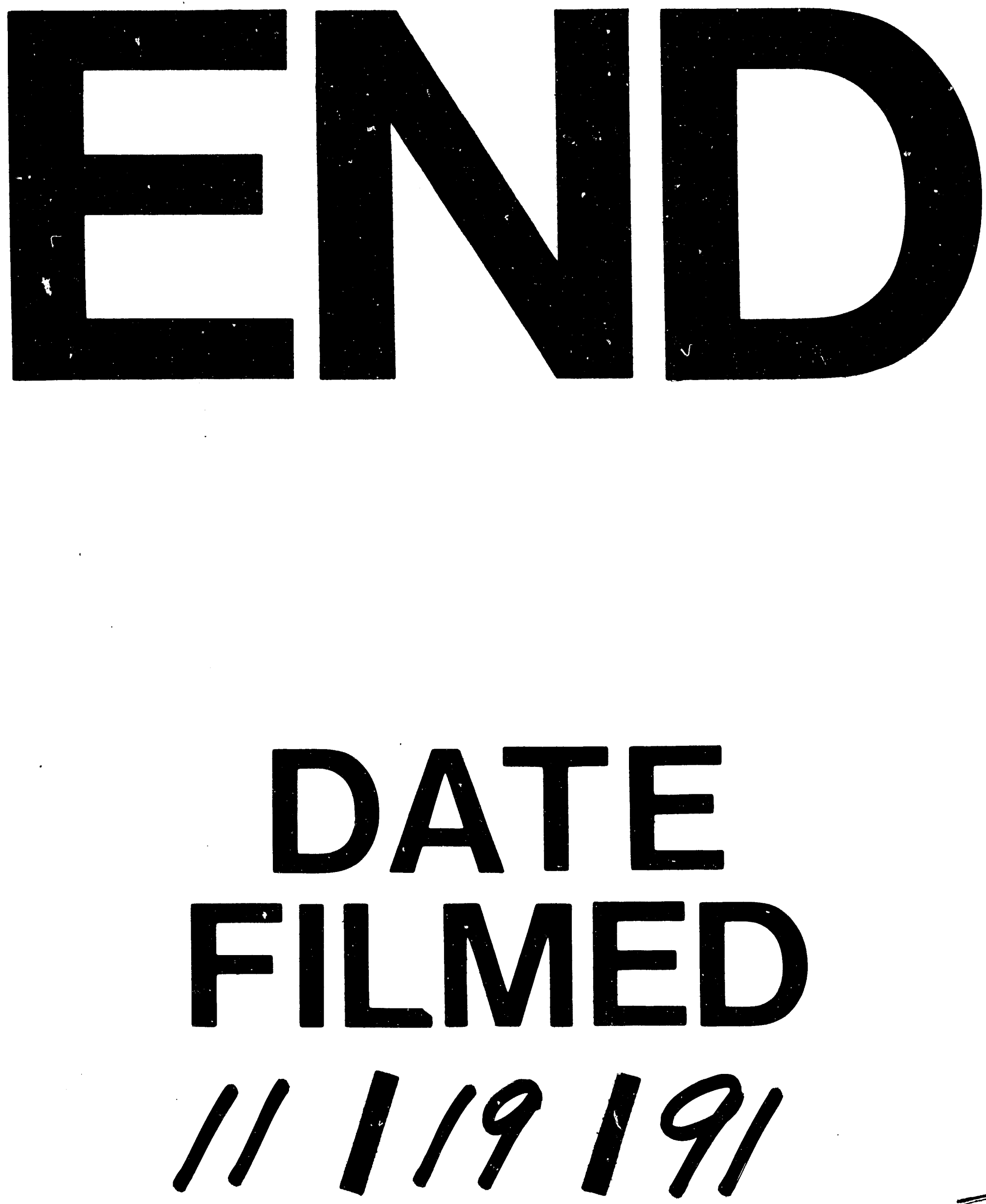

II 